\title{
FTM-based Simulation and Evaluation of Apparent Reduction in Elastic Modulus
}

\author{
S Ihara1*, M Uematsu', T Hasebe ${ }^{2}$ \\ 1. Graduate School of Kobe University, Japan \\ 2. Kobe University, Japan
}

\begin{abstract}
This study attempts to quantitatively evaluate the apparent reduction in elastic moduli, generally observed in metallic materials during unloading and/or cyclic elasto-plastic deformation, based on Field Theory of Multiscale Plasticity (FTMP). Two typical arrangements of bowing-out dislocation segments that yield mutually-distinct trends are examined by utilizing the FTMP-based duality diagram representation scheme. It is demonstrated that not only the scheme allows us to visualize the associated energy flow but also to correlate the resultant apparent shear modulus reduction rate in a unified manner by the duality coefficient measured on the duality diagrams. The scheme is demonstrated to be effective also for more complex cases accompanied by pinning/unpinning and the following relaxation processes.
\end{abstract}

\section{INTRODUCTION}

Dislocation is a major carrier of plasticity in crystalline solids, which is often compared to "wrinkle" of a wide-spreading carpet as a tangible simile. Although dominant roles of dislocations have been well-recognized and well-documented to date, still there seem to be many mysteries remain unfolded, e.g., with respect to their critical roles in controlling micro/macroscopic properties of the targeted crystalline materials. One of the reasons is that dislocations do not always determine the system response individually but frequently do as groups, like as substructures evolving during the course of elasto-plastic deformation. One of such eloquent examples is "dislocation cells" that are universally observed in plasticallydeformed crystals [1] [2], in the sense that they substantially controls the overall mechanical properties notwithstanding we do not essentially know why and how they are formed. Since the dislocation cells are composed of extremely large number of dislocations having roughly a periodic structure with wavelengths commensurate with submicron to micron, which is much larger than the magnitude of Burgers vector (characteristic (intrinsic) length of dislocation), it may safely be said that this dilemma is a critical "missing link" against achieving multiscale modeling of plasticity in terms of "transcending” scales.

${ }^{*}$ Corresponding Author: ihara.shirou@mail.mm4.scitec.kobe-u.ac.jp 
To solve the above dilemma, we ought to establish, above all, how to view dislocation aggregates in coarse-grained manner, maintaining their linear nature including the configurational complexities when necessary. Figure 1 shows a schematic drawing for "discrete vs. continuum" dislocations in 2D and 3D. One can notice exclusive difficulties in expressing 3D discrete dislocations based on a continuum picture, whereas relatively easy method may be found for 2D counterparts such as using distribution functions like for aggregates of particles. We must admit almost nothing has been done in this respect since Kröner suggested a use of statistical mechanics in his approach based on multi-point dislocation correlation functions for expressing distributed dislocations [3].

We have advocated a generic theoretical framework called FTMP (Field Theory of Multiscale Plasticity) [4] [5] [6] [7], together with the associated evaluation schemes, with which we successfully demonstrated to be able to capture the evolutionary aspects of dislocation ensembles, both qualitatively and quantitatively, as evolving inhomogeneities. Based on FTMP, we tackled the above problem in several respects, e.g., collapsing mixed dislocation walls [8] and GNBs (Geometrically Necessary Boundaries) [9], against simulated results by using discrete dislocation dynamics (DD) [10] [11], where dynamic behaviors of wall-constructing dislocation networks are successfully treated in a unified manner, particularly, by utilizing the FTMP-based duality diagram representation scheme. In this paper, we take afresh up another sort of dynamic behaviors of discrete dislocations that leads to softening of macroscopic stress responses of material systems, i.e., apparent reduction in the elastic (shear) modulus.

Experimentalists might have noticed decreasing elastic modulus observable in loading and reloading stress-strain curves (e.g., [12] [13]), implying the elastic modulus (e.g., Young's modulus) is not always be constant at least apparently during elasto-plastic deformation. This phenomenon causes many engineering problems, greatly affecting the predicting precisions of practically-important mechanical properties of metallic materials such as spring back and the amount of ratcheting [14]. Few studies, however, seem to have been carried out (this phenomenon seem to have not been taken so seriously by analytical researchers) probably and partially because of lack of such practical experiences.

The simplest mechanism yielding the "apparent" reduction in the elastic modulus is damping motions of pinned dislocation segments [15] [16]. During loading and unloading, pinned dislocation segments bow out and subsequently come back to the original configurations if there is no internal stress. This "reversible" plastic response consequently contributes to the "apparent softening" in the elastic stress-strain response, which appears as the apparent "reduction in the shear modulus."

This study extensively discusses the "apparent reduction in the shear modulus" based on FTMP by focusing on damping motions of dislocation segments via their bowing-out movements, against a series of simulated dislocation systems by using DD. Starting from a simple set of configurations of pinned segments of dislocations, we also extend the discussion to more complex conditions accompanied by their unpinning and relaxation processes. 


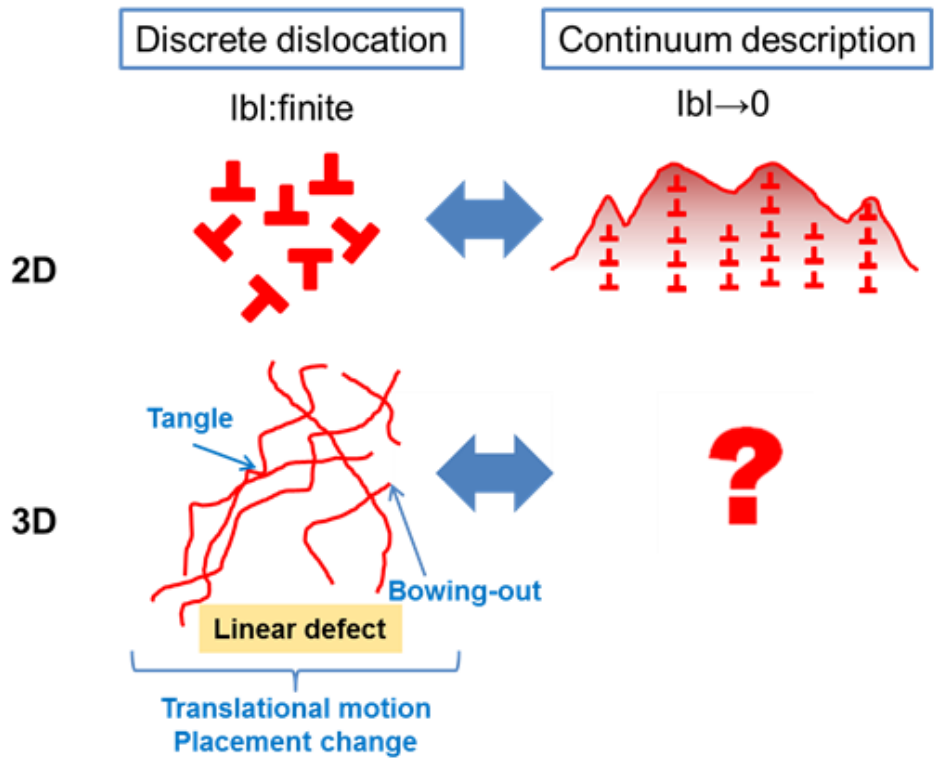

Figure 1. Discrete vs. continuum representations of dislocation aggregates.

\section{ABOUT “APPARENT” REDUCTION IN ELASTIC MODULUS}

Let $\Delta \gamma^{p}$ be the recovered plastic strain and $\Delta \mu$ be the apparent decrement of the shear modulus caused by $\Delta \gamma^{p}$, we have,

$$
\gamma^{e}+\Delta \gamma^{p}=\left(\mu^{-1}+\Delta \mu^{-1}\right) \tau=\mu^{-1}\{1+(\mu / \Delta \mu)\} \tau
$$

What we resultantly observe as the shear modulus from the given stress-strain response is $\mu\{1+(\mu / \Delta \mu)\}^{-1}$ instead of $\mu$. Therefore, the "apparent" reduction ratio of the shear modulus is given by,

$$
\mu^{\prime} / \mu \equiv\{1+(\mu / \Delta \mu)\}^{-1}
$$

where $\mu^{\prime}$ denotes the shear modulus to be observed.

The above $\Delta \gamma^{p}$ in Eq. (1) is derived in a way presented in Figure 2, where a pinned dislocation segment under an applied shear stress $\tau$, is schematically shown, with bowed-out curvature of $1 / R=\tau b / T$ and the corresponding swept-out area of $\Delta A$. Here, the line tension $T$ is approximately given by $T \approx \mu b^{2} / 2$. The reversible plastc shear increment produced by the bowing is,

$$
\Delta \gamma^{p}=b \Delta A / V
$$




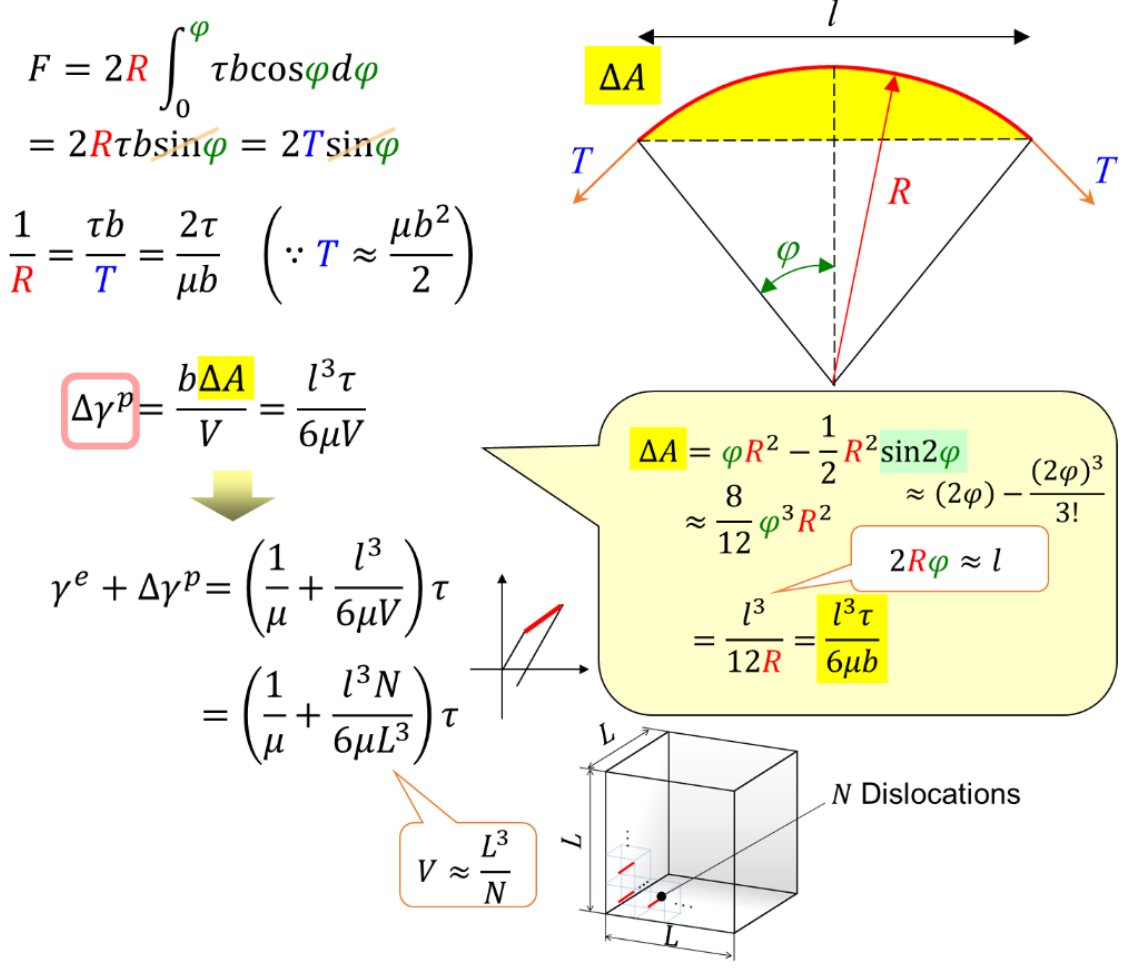

Figure 2. Schematic drawing of derivation process for deriving the attendant apparently reduced shear modulus due to a bowing-out dislocation segment.

where by small bow out

$$
\Delta A=\varphi R^{2}-\left(R^{2} \sin 2 \varphi\right) / 2
$$

Expansion of the $\sin 2 \varphi$ by small arguments gives,

$$
\Delta A=8 R^{2} \varphi^{3} / 12
$$

but since by small bow out $2 \varphi R \approx l$,

$$
\Delta A=l^{3} /(12 R)
$$

Going upon substitution, we finally have,

$$
\Delta \gamma^{p}=l^{3} \tau /(6 \mu V)
$$


Since $\Delta \gamma^{p}$ is reversible, it will masquerade as an added elastic strain giving an apparent reduced shear modulus, as argued above. Substituting this $\Delta \gamma^{p}$ into Equation (1), considering $\mathrm{N}$ dislocations in a cube with the edge length $\mathrm{L}$, we ultimately have,

$$
\mu / \mu^{\prime}=\left\{1+l^{3} N /\left(6 L^{3}\right)\right\}^{-1}
$$

\section{ANALYTICAL MODEL AND PROCEDURE}

\subsection{FTMP-based Incompatibility Model and Duality Diagram Representation}

Given 3D dislocation configurations, the dislocation density tensor $\alpha_{i j}$ is firstly evaluated for each sub-cell, where the simulation cell is divided into $100 \times 100 \times 100$ sub-cells in the present study. Here, a coarse-grained line vector is introduced for the dislocations contained in each sub-cell, without which all the details about the segment-wise geometrical fluctuations affect the distinction between edge and screw components. The definition of $\alpha_{i j}$ in this context is given as [17],

$$
B_{i}=\alpha_{j i} d S_{j}
$$

where $B_{i}$ is the Burgers vector corresponding to the coarse-grained line vector and $d S_{j}$ is the area through which the dislocations penetrate. Note the total length of the dislocations within the sub-cell is renormalized into the coarse-grained counterpart to conserve the density.

Based on the thus evaluated dislocation density tensor defined in Equation (9), the incompatibility tensor is further evaluated as [18],

$$
\eta_{i j}=-\left(\epsilon_{i k l} \partial_{k} \alpha_{j l}\right)_{S Y M}
$$

where the spatial derivative is evaluated for a given arbitrary coarse-grained region by utilizing the least-square method [19].

According to the flow-evolutionary hypothesis [7] in FTMP, the incompatibility tensor is equated with the fluctuation part of the energy-momentum tensor, where both the tensors are defined in the four-dimensional space-time. The temporal components provide the following specific relationship (assuming static conditions),

$$
\eta_{K K}=\kappa \delta U^{e}
$$

where $\kappa$ is named duality coefficient. Based on Equation (11), we can draw duality diagrams, i.e., the relationship between the trace of the incompatibility tensor and (the fluctuation part of) the elastic strain energy. The duality diagram representation allows us to "visualize" the energy conversion process or "energy flow" in terms of dislocation movements within the system. 


\subsection{Analytical Conditions and Simulation Models}

We perform a series of dislocation dynamics simulations [11] for obtaining dislocation systems to be examined. Figure 3 shows the simulation cell with $(2000 \mathrm{~b})^{3}$ edges, together with an edge dislocation segment on a prescribed slip plane. We assume $\alpha$-Fe (BCC), with the density $7.88 \times 10^{3} \mathrm{~kg} / \mathrm{m}^{3}$, the shear modulus $80.0 \mathrm{GPa}$, Poisson's ratio $v=0.324$, and the magnitude of Burgers vector $b=2.483 \times 10^{-10} \mathrm{~m}$. The external shear stress $\tau$ is applied as shown in Figure 3 until 500 steps (loading) and is then reversed until 1,000 steps (unloading), with the increment of the simulation time step being $1.8 \times 10^{-12} \mathrm{sec}$.
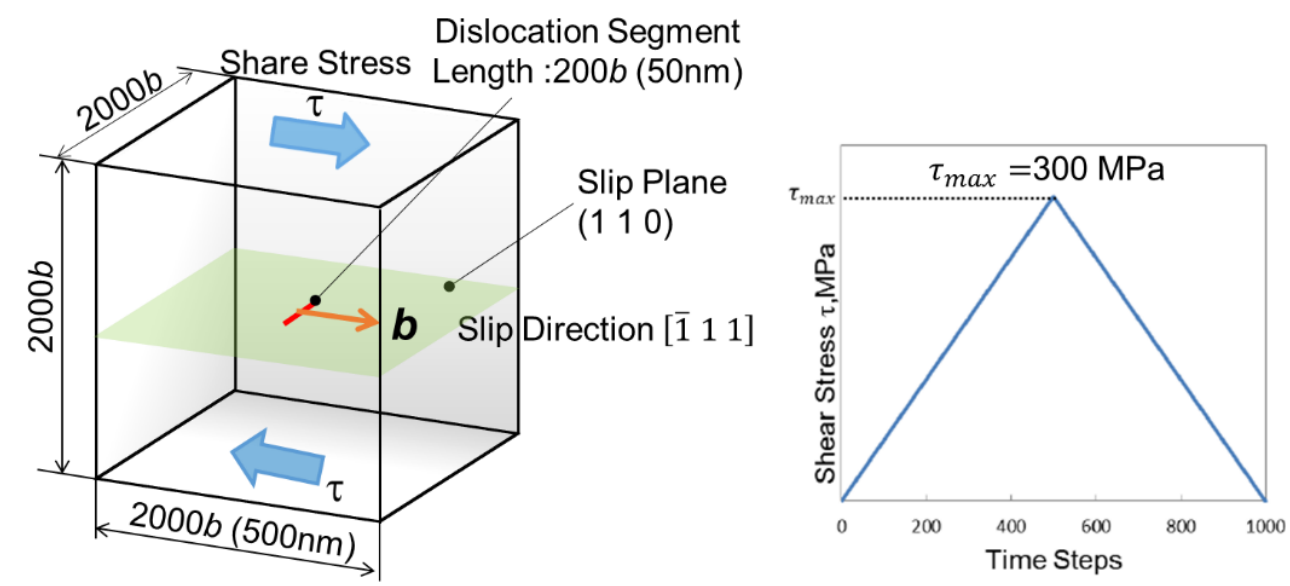

Figure 3. Schematic drawing of simulation cell and loading condition.

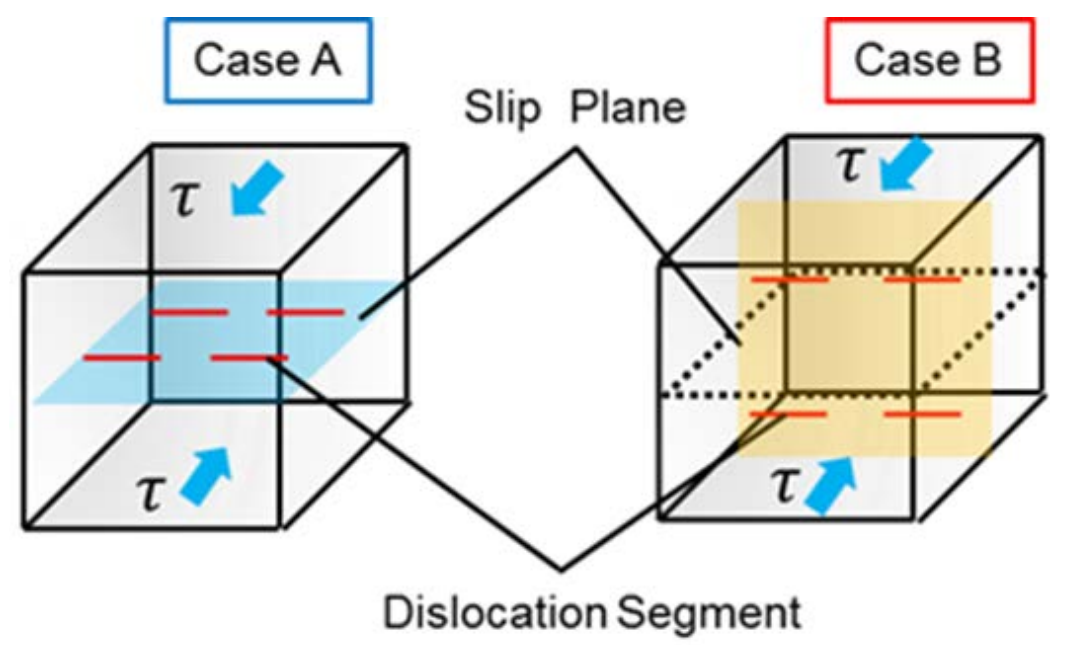

Figure 4. Schematics of simulation models with two representative dislocation arrangements, Case A (horizontal arrangement) and Case B (vertical arrangement). 
Pinned dislocation segments are placed in the simulation cell as schematically shown in Figure 4 , where the initial length of each segment is set to be 200b (b is the magnitude of Burgers vector). We consider roughly two arrangements of the dislocation segments, as shown in the figure, i.e. horizontal (Case A) and vertical (Case B) arrangements with respect to the slip plane. Note the Case B (vertical arrangement) may be regarded as a simplified model for dislocation walls. In this study, we arrange the edge dislocations so that the number of dislocations $\mathrm{N}=45,75,125,175,225$ and 325 for both the cases.

\section{RESULTS AND DISCUSSIONS}

4.1. Stress-strain response and apparent reduction in shear modulus

Figure 5(a) shows the simulation set ups for Cases A and B. We also present the upper view for $\mathrm{N}=175$ in Figure 5(b) as a representative case, showing bowing-out dislocation segments. As observed in these snapshots, we immediately notice that the bowing-out motions are greatly restricted in Case B compared with those in Case A, probably because of the induced back stress field by themselves.

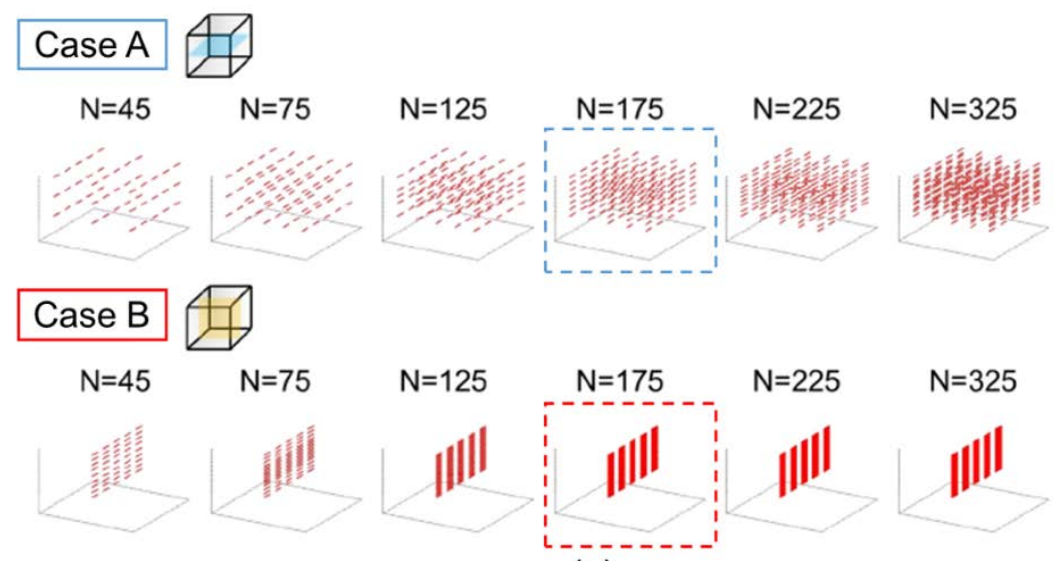

(a)

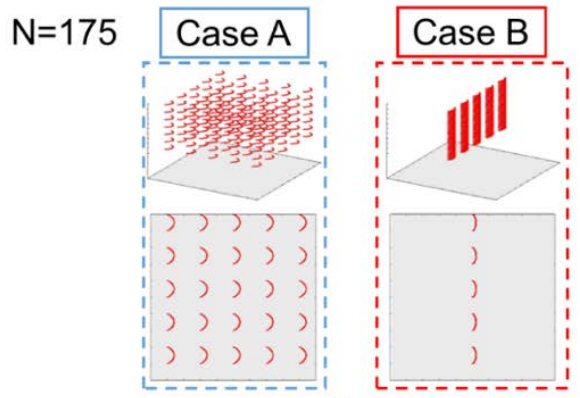

(b)

Figure 5. Snapshots of (a) initial configurations of whole models and (b) bowingout dislocation segments in the case of $\mathrm{N}=175$ for Cases $\mathrm{A}$ and $\mathrm{B}$. 
Figure 6(a) and (b) displays the corresponding variations of shear stress-shear strain curves with the number of dislocation segments to Figure 5 for Cases A and B, respectively. First thing that we can confirm is the decreasing slopes of them with increasing number of dislocation segments for both the cases. The second, on the other hand, is the much smaller slopes for Case B than those for Case A, reflecting the restricted bowing-out motions of the dislocation segments demonstrated in Figure 5. Figure 6(c) compares the representative shear stress-strain curves between the two cases, by taking a representative condition, i.e., $\mathrm{N}=175$. Both the cases, however, exhibit nearly "reversible" stress-strain responses; the plastic shear strain caused by the bowing-out motions of dislocation segments is roughly recovered to zero when the segments come back to the original configuration (i.e., straight line).

The corresponding "apparent" reduction ratios of the shear modulus to the results in Figure 6 are calculated by using Equation (2). Figure 7 shows variations of the "apparent" reduction rate of the shear modulus with increasing number of dislocation segments $\mathrm{N}$, comparing Cases A, B and the theory given by Equation (3) (where L=2000b and l=200b are substituted). The results for Case A agree well with the theory, whereas Case B exhibits deviation from the theory as $\mathrm{N}$ increases. The deviation corresponds to the greatly-restricted bowing-out motions of dislocation segments seen in Figure 5(b).

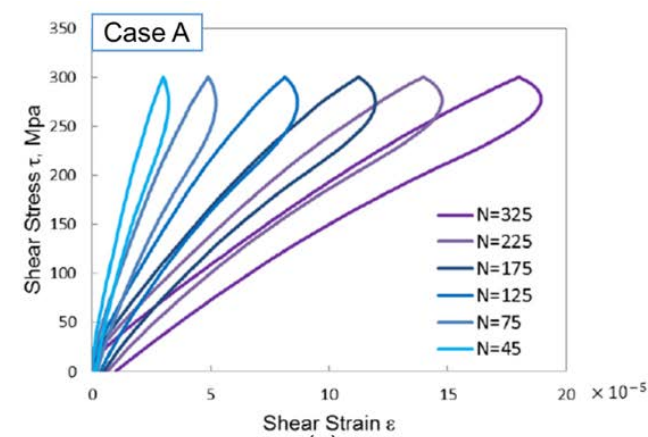

(a)

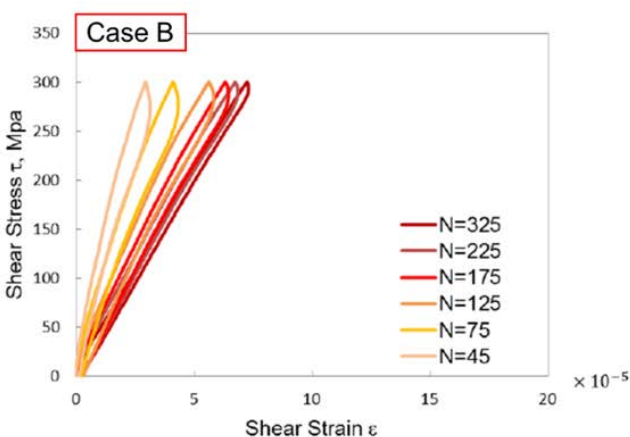

(b)

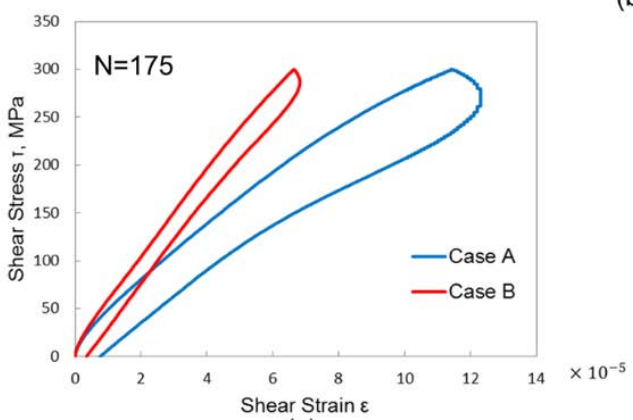

(c)

Figure 6. Shear stress-plastic shear strain responses for (a) Cases A, (b) B and (c) both cases in the case of $\mathrm{N}=175$. 


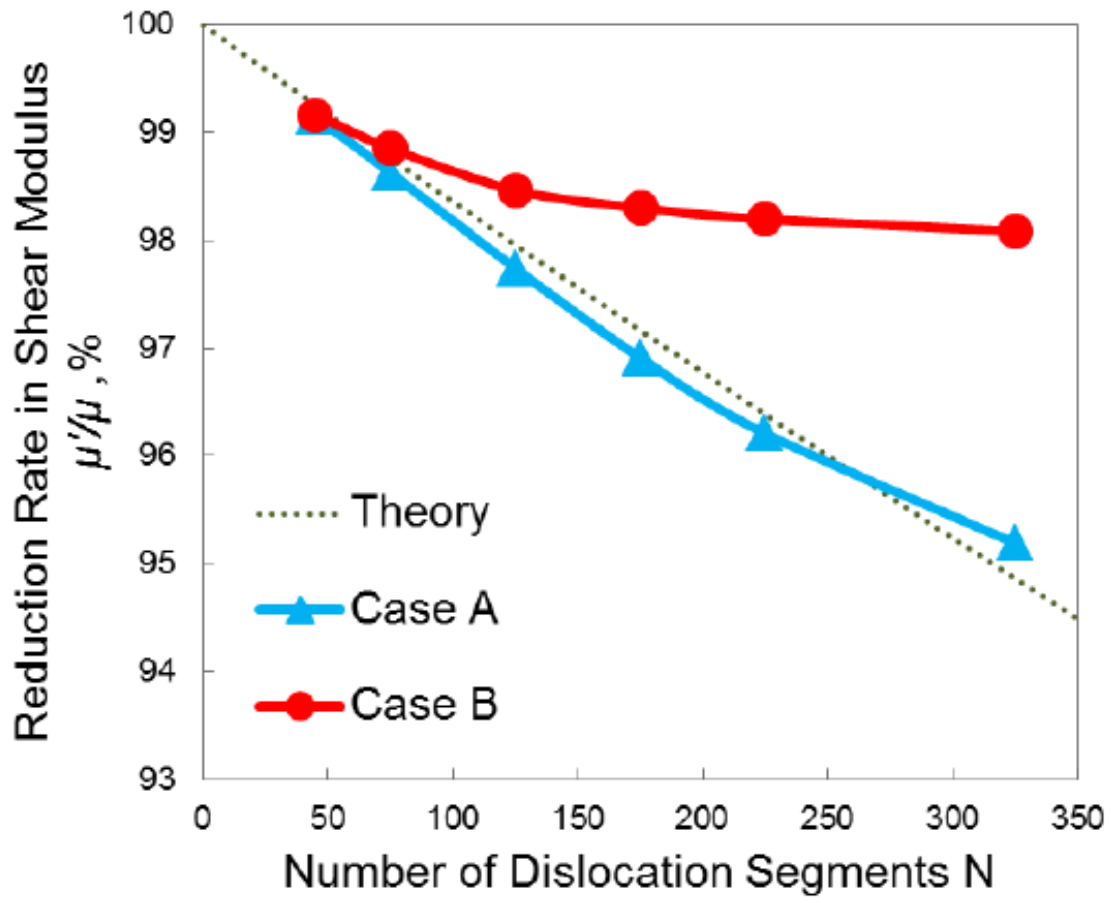

Figure 7. Relationship between "apparent" reduction rate in shear modulus and the number of dislocation segments.

\subsection{Duality diagram representation}

We examine the above bowing-out responses of the dislocation segments by utilizing the duality diagram representation scheme, based on the flow-evolutionary hypothesis [7]. Here, the incompatibility tensor and the elastic strain energy are calculated by Equation (5) and the stress field of dislocations, respectively. Figure 8(a) compares duality diagrams for all the conditions of the number of dislocation segments $\mathrm{N}$ between Cases $\mathrm{A}$ and $\mathrm{B}$, whose representatives $(\mathrm{N}=175)$ are detailed in Figure $8(\mathrm{~b})$, respectively, while the extracted plots from (a) at the maximum stress (500steps) are shown in Figure 8(c). As demonstrated in Figure 8(b), Case A exhibits a sharp increase and the subsequent decrease in $\eta$ almost vertically in the diagram, meaning most of the externally-applied work is consumed as the incompatibility grows, without being stored in the dislocations. In sharp contrast, Case B tends to yield the horizontal growth in $\eta$, i.e., the growth rate saturates rapidly as the elastic strain energy increases, indicating that the external energy is effectively stored in the bowing-out dislocation segments until the critical configuration is reached. 


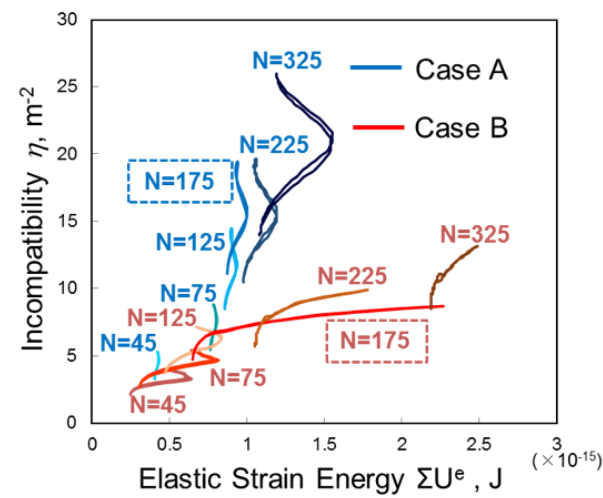

(a)

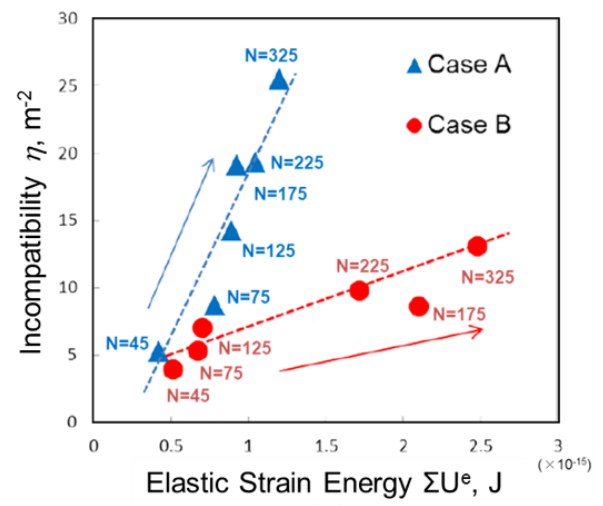

(c)

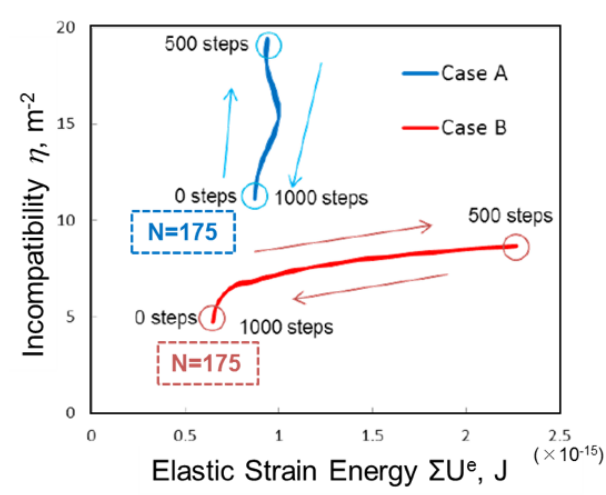

(b)

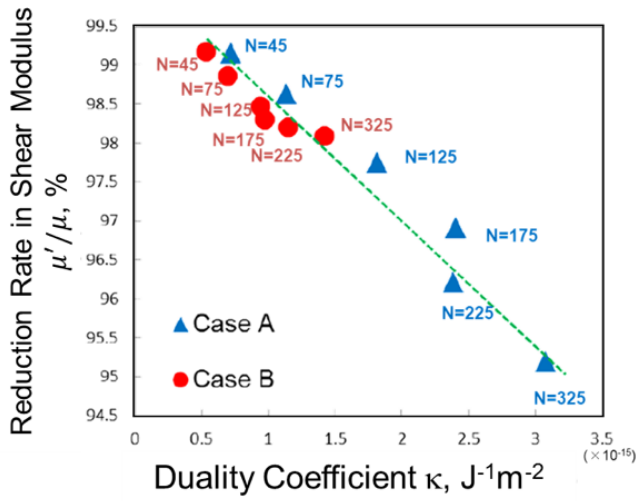

(d)

Figure 8. Duality diagrams comparing Cases A and B, (a) through (c), together with correlation of apparent reduction rate of shear modulus with duality coefficient, (d).

The above distinct trends between Cases A and B well correspond to those in the bowingout configurations of dislocation segments in Figure 5. Thus way, the energy flow associated with the configurational changes of the dislocations can be visualized via the duality diagram.

Regarding the extracted plots in Figure 8(c), we find linear relationships roughly holding between the incompatibility and the elastic strain energy for both the cases, respectively. The difference in the slopes is attributed to that in the energy conversion characteristics between the two cases discussed above: the large slope for Case A represents large energy conversion rate to the incompatibility-related degrees of freedom, whereas the small slope for Case B indicate the small conversion rate. 
For evaluating this quantitatively, we further evaluate the duality coefficient via $\kappa \equiv$ $\sum \eta / \sum U^{e}$ from Figure 8(c), which measures how much strain energy is converted to, i.e., dissipated into, local plasticity, i.e., the bowing-out motions of dislocation segments, in the present case, that manifests itself as the growth of the incompatibility tensor field. Figure 8(d) correlates the apparent reduction rate of the shear modulus with $\kappa$. There observed a good correlation between the two, regardless of not only the arrangements (Cases A and B) but also the number of dislocation segments, strongly implying the duality coefficient can be a parameter measuring the system response for the present dislocation systems in terms of the above-mentioned energy conversion characteristics.

We extend the simulation set-up to more complex situation, where dislocation segments are rather randomly distributed. To this end, we make use of the model with distributed precipitates employed in [20]. Repulsive interactions against the precipitate models is assumed with $\mathrm{N}=100$ and 400 . Obtained results are compared in Figure 9, yielding a certain amount of "non-recoverable" shear strain due to occasional unpinning of the segments for both the cases. For a quantitative discussion with respect to the relationship between the mean pinning space and the irreversible stress-strain behavior, we measure the "pinning space" distribution, and further fabricate five simplified models as displayed in Figure 10 based on it.
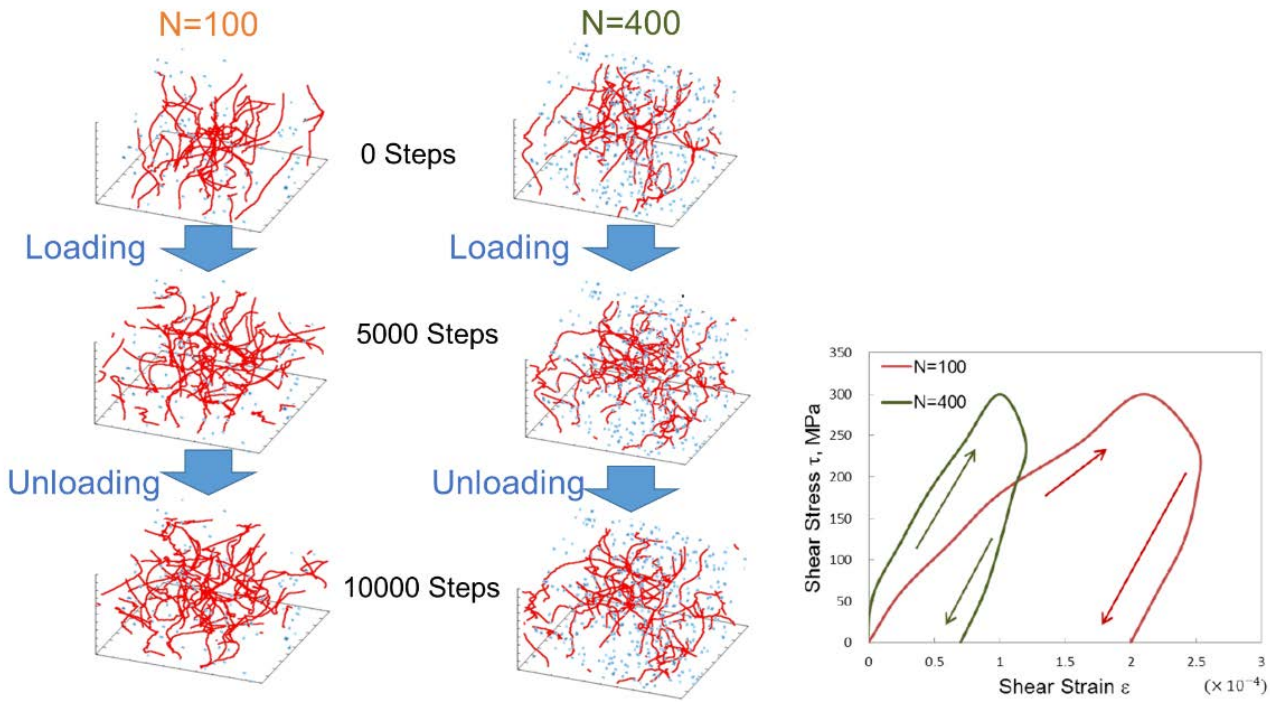

Figure 9 Series of snapshots during loading and unloading processes for models with randomly-distributed precipitates (pinning points) [20], comparing two cases with $\mathrm{N}=100$ and 400 (left), together with the corresponding system-wise shear stress-strain curves (right). 


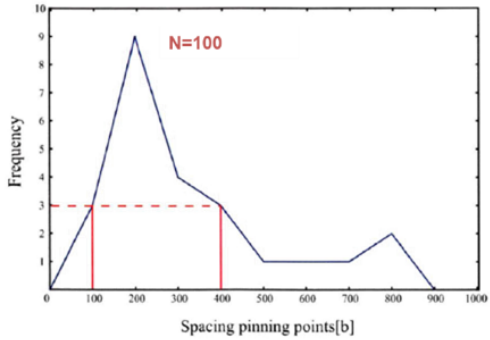

(a)
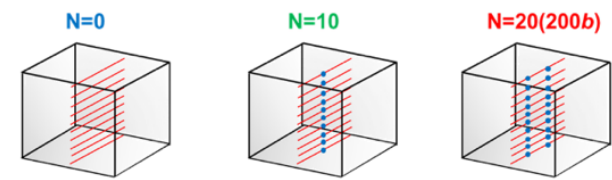

$800 \mathrm{~b}$

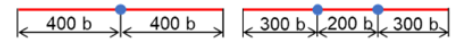
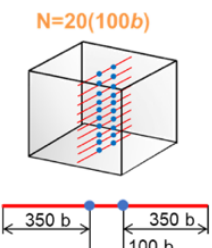

$100 \mathrm{~b}$

Figure 10 Pinning space distribution (left) of dislocation systems in Figure 9, together with schematics of simplified models based on it (right).

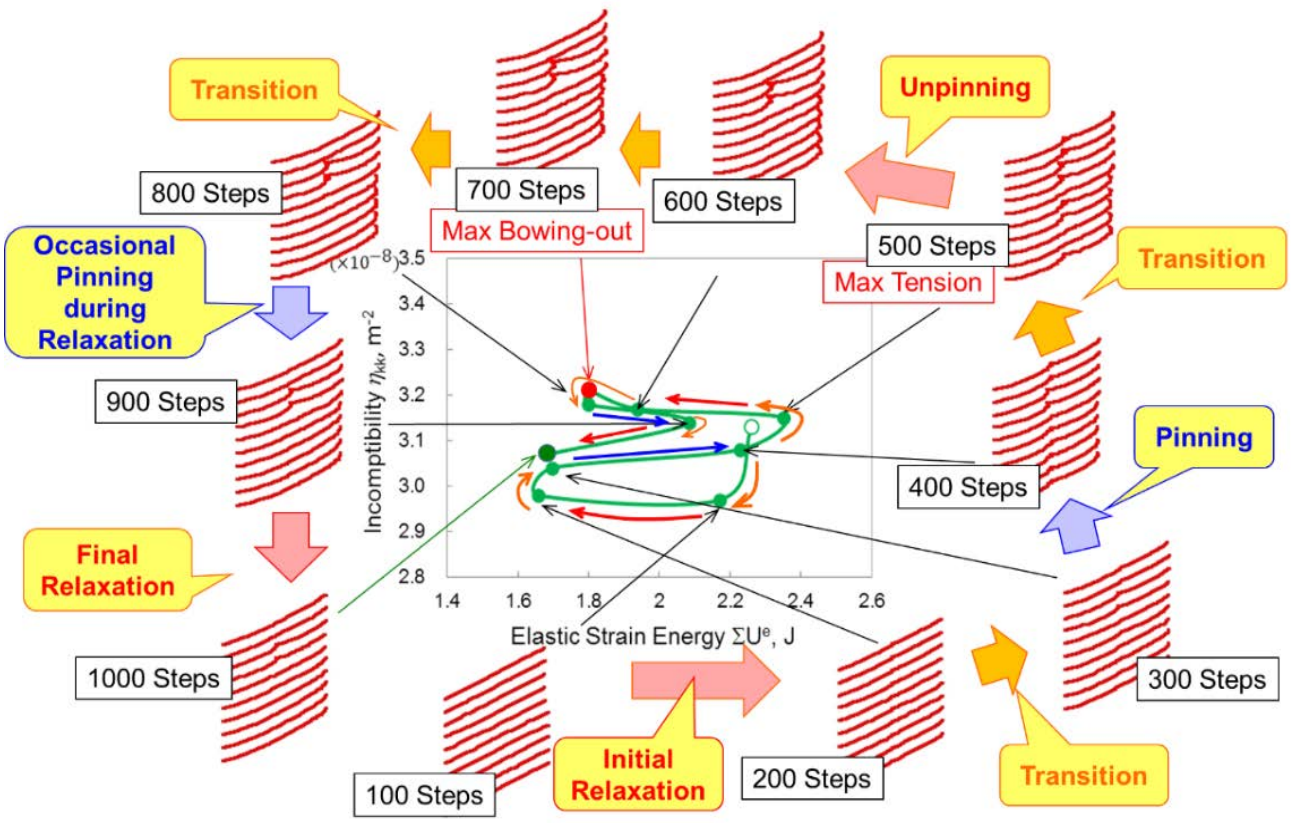

Figure 11 A typical duality diagram obtained for simplified models, together with snapshots at representative steps in the case of $\mathrm{N}=10$, where precipitates in Figure 10 are not shown. 
As representative case, some details of the duality diagram for the model with $\mathrm{N}=10$ are presented in Figure 11, with snapshots at representative steps. In the duality diagram, roughly three processes are involved, in addition to the initial and final relaxation steps, i.e., pinning and unpinning processes, each manifested as "positive" and "negative" growths, respectively, together with transitions that bridge the two. The maximum bowing-out configuration is shown to be reached at 700 steps, 200 step-after the maximum tension at 500 steps due to the inertial effect.

Figure 12 displays all the duality diagrams including that for $\mathrm{N}=0$. We observe larger incompatibility range as the number of precipitates increases and/or the inter-precipitate spacing decreases, otherwise roughly the same trend as that for $\mathrm{N}=10$ in Figure 11, demonstrating basically the same dislocation behaviors during the process, except the case with $\mathrm{N}=0$.

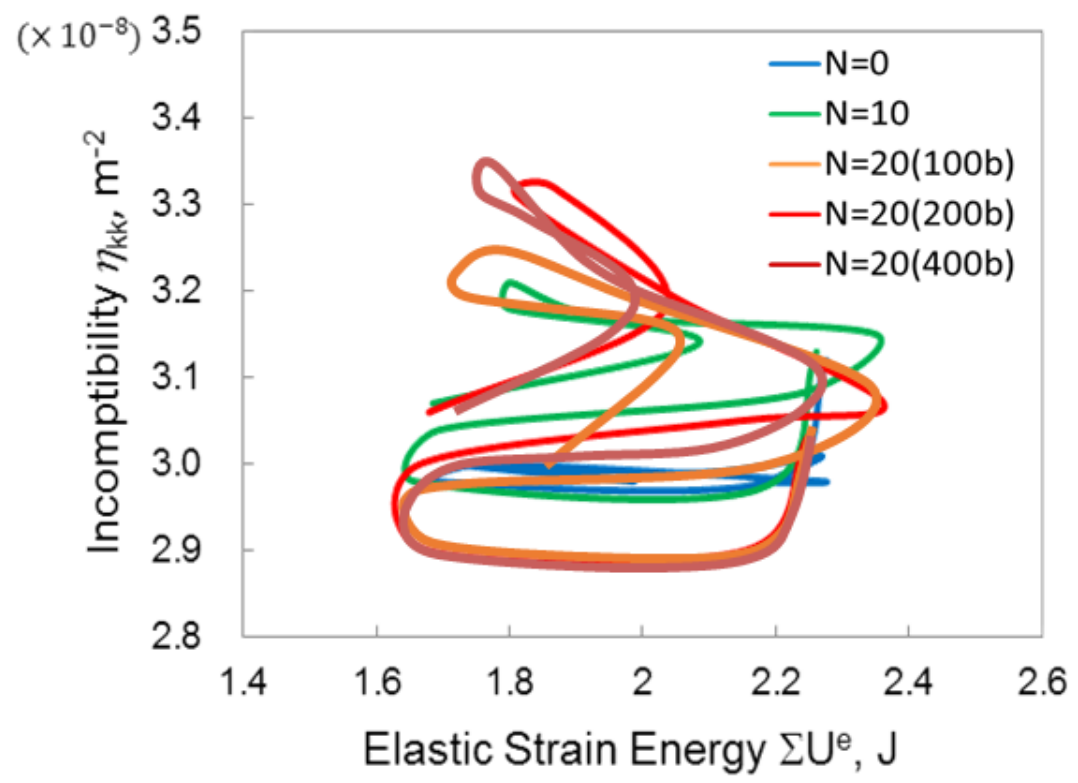

Figure 12 Comparison of duality diagrams among five simplified models in Figure 10.

The corresponding stress-strain curve is displayed in Figure 13 to Figure 11 for $\mathrm{N}=10$, comparing with those for other conditions. Clearly confirmed first is the deviation from the reference $(\mathrm{N}=0)$ are started to occur at 300 steps upon unpinning, whose trend is in common to all the models. That confirmed second is the unloading response is characterized by the slope right before the final relaxation between 800 and 900 steps, which occasional pinning takes place during the transition process. 


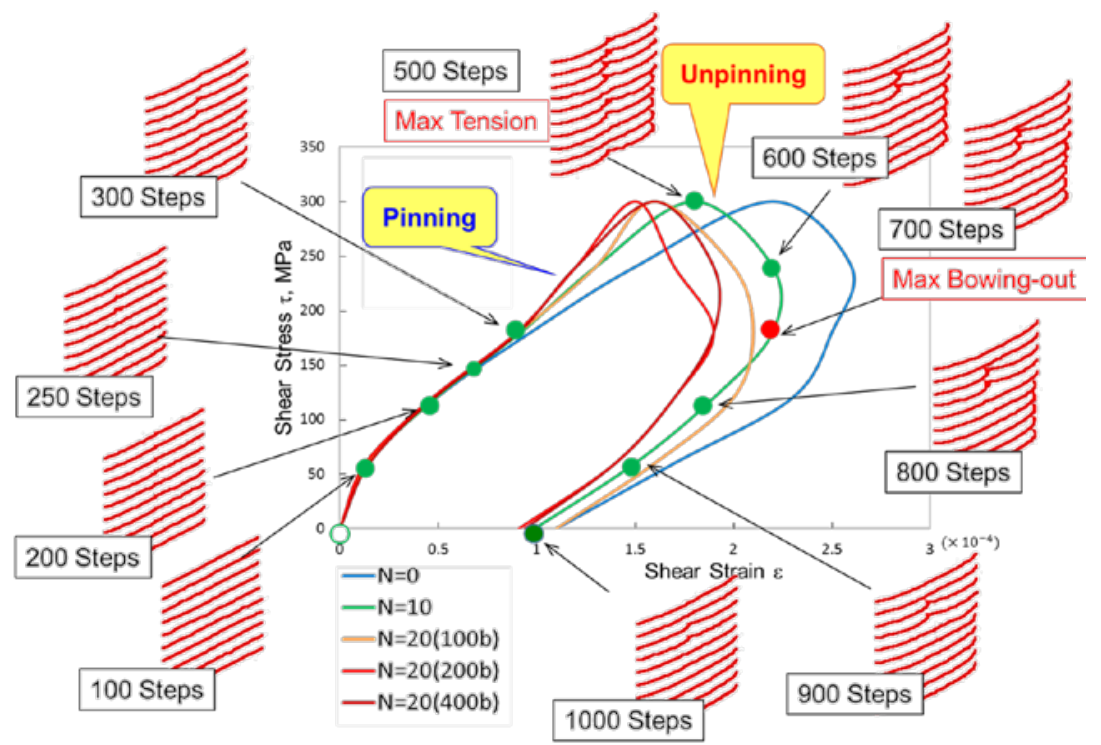

Figure 13 Stress-Strain curves comparing five simplified models in Figure 10, together with snapshots at representative steps for $\mathrm{N}=10$.
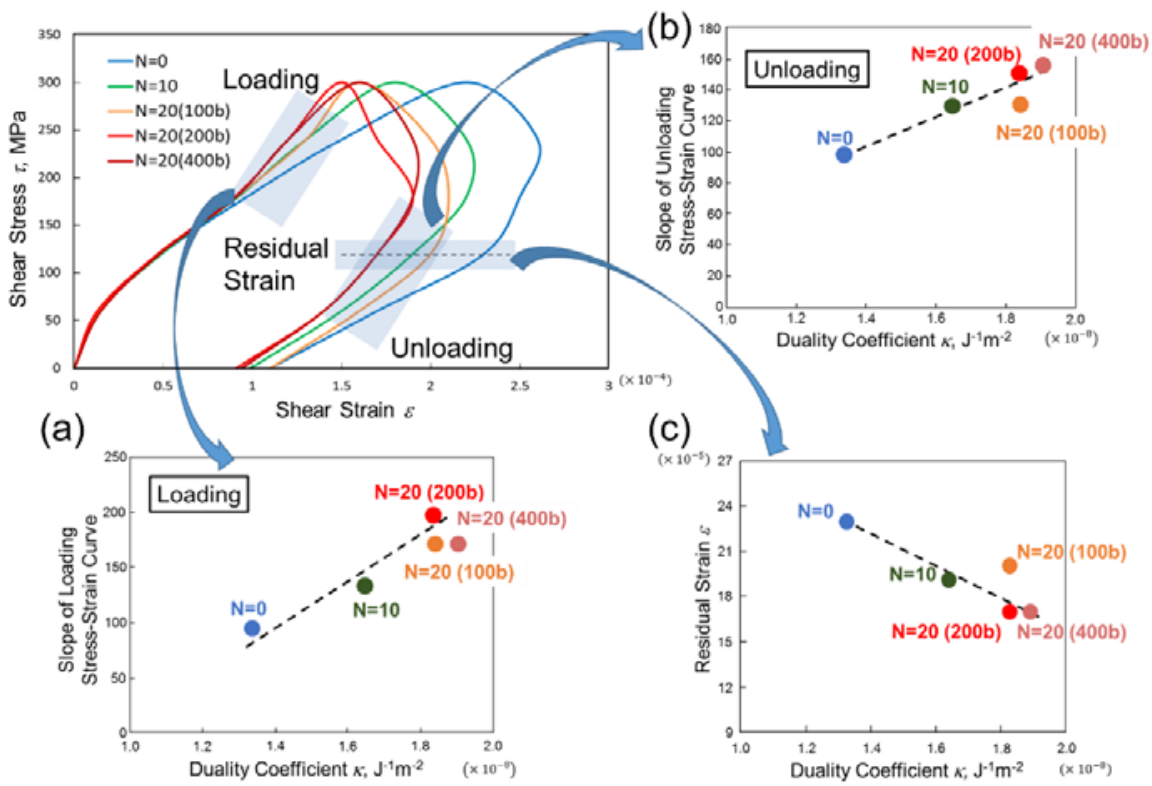

(a)

(c)

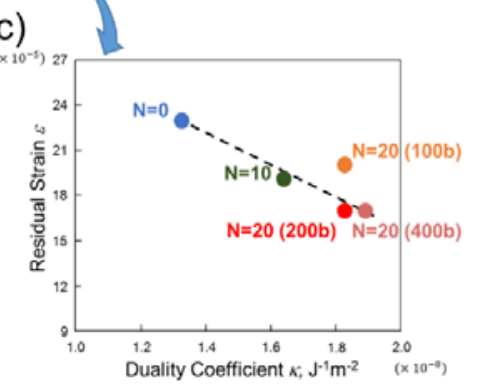

Figure $14 \kappa$-based correlations of (a) loading slope, (b) unloading slope and (c) residual strain for five simplified models, together with system-wise stress-strain curves (redisplayed). 
Figure 14 shows the results of comparing the duality coefficients and the quantity which characterize the stress-strain curves, i.e., residual strain and slopes at loading and unloading steps, for the five simplified models. As representative evaluation results, we present correlations of two representative slopes as confirmed above, together with the residual strain with the duality coefficient. Since satisfactory correlations are obtained regardless the models, applicability of the method presented in the paper to more complex situations are demonstrated.

\section{CONCLUSION}

This study described apparent reduction in elastic moduli often observed empirically in metallic materials during plastic deformation due to damping motions of dislocation segments from the viewpoint of FTMP (Field Theory of Multiscale Plasticity). Bowing-out configurations of dislocation segments for two typical dislocation arrangements, i.e., those aligned on the horizontal and the vertical plane to the imparted shear stress, were simulated by discrete dislocation dynamics, showing mutually-distinct trends: The vertical arrangement exhibits greatly restricted bowing-out motions compared to the horizontal counterparts, resulting in relatively smaller reduction ratio. The obtained results were extensively examined by FTMP-based duality diagram representation scheme, which allowed not only to visualize the associated energy flow into the incompatibility-related degrees of freedom for each case, but also to quantitatively correlate the apparent reduction rate of the shear modulus in a unified manner via the duality coefficient.

We also made an extended application of the above evaluation scheme to more complex conditions, where dislocation segments are pinned by a number of precipitates that yield relatively complicated behaviors including pinning/unpinning events during the imparted loading-unloading process. It was shown that basically the duality diagram-based scheme is applicable to them, leveraging the validity of the present FTMP-based approach.

\section{REFERENCES}

[1] L. P. Kubin, "Dislocation Patterns: Experiment, Theory and Simulation," in Stability of Materials, A. Gonis, P. E. A. Turchi and J. Kudrnovský, Eds., Plenum Press, New York, 1996, pp. 99-135.

[2] S. Raj and G. M. Pharr, "A compilation and analysis of data for the stress dependence of the subgrain size," Mater. Sci. Eng., vol. 81, pp. 217-237, 1986.

[3] E. Kröner, "Initial Studies of a Plasticity Theoy Based upon Statistical Mechanics in Inelastic Behavior of Solids," in Inelastic Behavior of Solids, M. F. Kanninen, W. F. Adler, W. F. Rosenfield and R. I. Jaffee, Eds., McGraw-Hill, New York, 1970, pp. 137147.

[4] T. Hasebe, "Continuum Description of Inhomogeniously Deforming Polycrustalline Aggeregate ased on Field Theory," IUTAM Symposium on Mesoscopic Dynamics of Fracture Pricess and Materials Strength. Edited by kitagawa, H. and Shibutani, Y., Kluwer Academic Publishers, pp. 381-390, 2004.

[5] T. Hasebe, "Interaction Fields Based on Incompatibility Tensor in Field Theory of Plasticity -Part I: Theory-," IMMIJ, vol. 2, no. 1, pp. 1-14, 2009. 
[6] T. Hasebe, "Interaction Fields Based on Incompatibility Tensor in Field Theory of Plasticity -Part II: Application-," IMMIJ, vol. 2, no. 1, pp. 15-30, 2009.

[7] T. Hasebe, M. Sugiyama, H. Adachi, S. Fukutani and M. Iida, "Modeling and Simulations of Experimentally-Observed Dislocation Substructures Based on Field Theory of Multiscale Plasticity (FTMP) Combined with TEM and EBSD-Wilkinson Method for FCC and BCC poly/Single Crystals," Mater. Trans., vol. 55, no. 5, pp. 779-787, 2014.

[8] T. Hasebe and T. Naito, "FTMP-based 4D Evaluations of Discrete Dislocation Systems," in New Frontiers of Nanometals (Proc. 35th Ris $\varnothing$ int. Symp. on Maters. Sci.), S. Faester, Ed., 2014, pp. 305-312.

[9] S. Ihara and T. Hasebe, "FTMP-based Simulations and Evaluations of GeometricallyNecessary Boundaries (GNBs) of Dislocation," Int. Jnl. of Multiphysics, vol. 13, no. 3, pp. 253-268, 2019.

[10]L. Kubin, G. Canova, B. Devincre, V. Pontikis and Y. Brechet, "Dislocation Microstructure and Plastic Flow: 3D Simulation," Solid State Phenomena, vol. 23\&24, pp. 455-472, 1992.

[11]H. M. Zbib, M. Rhee and J. P. Hirth, "3D Simulation of Curved Dislocations: Discretization and Long range Interactions," in Advances in Engineering Plasticity and its Applications, T. Abe and T. Tsuruta, Eds., Pergamon, 1996, pp. 15-20.

[12] F. Yoshida, T. Uemori and K. Fujiwara, "Elastic-Plastic Behavior of Steel Sheets under in-plane Cyclic Tension-Compression at Large Strain," Int. J. Plast., pp. 633-659, 2002.

[13] M. Yang, Y. Akiyama and T. Sasaki, "Evaluation of Change in Material Properties due to Plastic Deformation," J. Mater. Process. Technol., pp. 232-236, 2004.

[14]R. H. Wagoner, "Sheet Springback," in Continuum Scale Simulation of Engineering Materials: Fundamentals - Microstructures - Process Applications, D. Raabe, F. Roters, F. Barlat and L. Chen, Eds., Wiley-VCH, 2004, pp. 757-774.

[15] N. Brown, "Observations of Microplasicity," in Microplasticity, C. J. McMahon, Jr., Ed., John Wiley and Sons Inc., New York, 1968, pp. 45-73.

[16] A. Granato and K. Lucke, "Theory of Mechanical Damping Due to Dislocations," J. Appl. Phys., vol. 27, pp. 583-593, 1956.

[17] J. F. Nye, "Some Geometrical Relations in Dislocated Crystals," Acta Metall., vol. 1, pp. 153-162, 1953.

[18]K. Kondo and M. Yuki, "On the Current Viewpoints of Non-Riemannian Plasticity Theory," in RAAG Memoirs of Unifying Study of Basic Problems in Engineering and Physical Science by Means of Geometry, vol. 2, K. Kondo, Ed., Gakujutsu Bunken Fukyu-kai, 1958, pp. 202-226.

[19] I. Yoon, "FTMP based Development of Continuous Field Evaluation Method for ThreeDimensional Discrete Dislocation System," Undergraduate Thesis, Kobe University, 2012.

[20] M. Yamada, T. Hasebe, Y. Tomita and T. Onizawa, "Dislocation Dynamics Simulation on Stability of High Dense Dislocation Structure Interacting with Coarsening Defects," IMMIJ, vol. 1, no. 4, pp. 437-448, 2008. 